\title{
Super-luminescent jet light generated by femtosecond laser pulses
}

SUBJECT AREAS:

NONLINEAR OPTICS

ULTRAFAST PHOTONICS

Received

23 September 2013

Accepted

7 January 2014

Published

27 January 2014

Correspondence and requests for materials should be addressed to

X.N.Z. (xnzhu1@ nankai.edu.cn)

\author{
Zhijun Xu, Xiaonong Zhu, Yang Yu, Nan Zhang \& Jiefeng Zhao
}

Institute of Modern Optics, Nankai University, Key Laboratory of Optical Information Science and Technology, Education Ministry of China, Tianjin 300071, P. R. China.

Phenomena of nonlinear light-matter interaction that occur during the propagation of intense ultrashort laser pulses in continuous media have been extensively studied in ultrafast optical science. In this vibrant research field, conversion of the input laser beam into optical filament(s) is commonly encountered. Here, we demonstrate generation of distinctive single or double super-luminescent optical jet beams as a result of strong spatial-temporal nonlinear interaction between focused $50 \mathrm{fs}$ millijoule laser pulses and their induced micro air plasma. Such jet-like optical beams, being slightly divergent and coexisting with severely distorted conical emission of colored speckles, are largely different from optical filaments, and obtainable when the focal lens of proper $\mathrm{f}$-number is slightly tilted or shifted. Once being collimated, the jet beams can propagate over a long distance in air. These beams not only reveal a potentially useful approach to coherent optical wave generation, but also may find applications in remote sensing.

ropagation of intense ultrashort laser pulses has encompassed many important nonlinear optical phenomena such as self-focusing ${ }^{1,2}$, supercontinuum generation ${ }^{3}$, conical emission ${ }^{4}$ etc. In particular, as a generic phenomenon, optical filaments have been extensively studied over the last three decades ${ }^{5-8}$. While the spatial and temporal aspects of Kerr effect and plasma related refractive index changes, interplaying with the linear dispersion and diffraction, are regarded as the primary mechanisms for light filament formation, other nonlinear effects including modulation instability ${ }^{9}$, self-steepening ${ }^{10}$, four-wave mixing ${ }^{11}$, third harmonic generation $^{12}$ and stimulated Raman scattering ${ }^{13}$ can also contribute to various characteristics of optical filaments.

One of the key parameters in characterizing a great majority of nonlinear interactions between an ultrashort laser pulse and the medium is the amount of overall nonlinear phase shift experienced by the propagating laser pulse. This nonlinear phase shift is normally compounded by the light-matter interaction strength and the effective interaction length. In practice, it is possible that the same amount of nonlinear phase shift can be resulted from either smaller refractive index change but a longer interaction length or a more significant refractive index change with a shorter interaction length. Optical filaments fall into the first category where both beam guiding and intensity clamping are present because of the delicate dynamic balance between Kerr lensing effect and defocusing effect of laser-induced plasma. In this regime initial laser beams are not or only weakly focused for most cases and hence the nonlinear refractive index change is limited to a relatively smaller value ${ }^{14}$. For tighter focusing, however, strong ionization at the focus can take place and this may effectively hinder the formation of optical filaments. Therefore, this leads to the second category of nonlinear interaction, namely, stronger nonlinear interaction but also shorter interaction length. For liquid or solid materials this regime of nonlinear interaction is often accompanied by optical breakdown and/or severe material damage ${ }^{15,16}$.

In this study, we report the observation of a novel phenomenon of the generation of brilliant jet light beams emanating from the micro air plasma induced by a relatively tight focused near-infrared femtosecond laser pulses $^{17,18}$. The single or double super-luminescent jet beam(s) (SJBs) that occur at the specific outskirt position of the severely distorted conical emission (CE) are obtained when the plano-convex focal lens of $100 \mathrm{~mm}$ focal length is properly shifted or tilted (See Methods). It is found that four-wave mixing (FWM) accompanied by beam symmetry breakup in CE is responsible for the formation of single and double SJBs.

\section{Results}

Generation of super-luminescent jet beams. Fig. 1a shows the schematic diagram for SJBs generation, in which $1 \mathrm{kHz}, 50$ fs laser pulses with $\sim 2.3 \mathrm{~mJ}$ pulse energy propagate right to left. (The two dashed arrow lines in Fig. 1a mark the usual diffraction if the incident pulse energy is low enough so that no air plasma is produced at the lens focus). In the case of ordinary air ionization, the focal lens on the right is placed normal, as well as centered, to the 

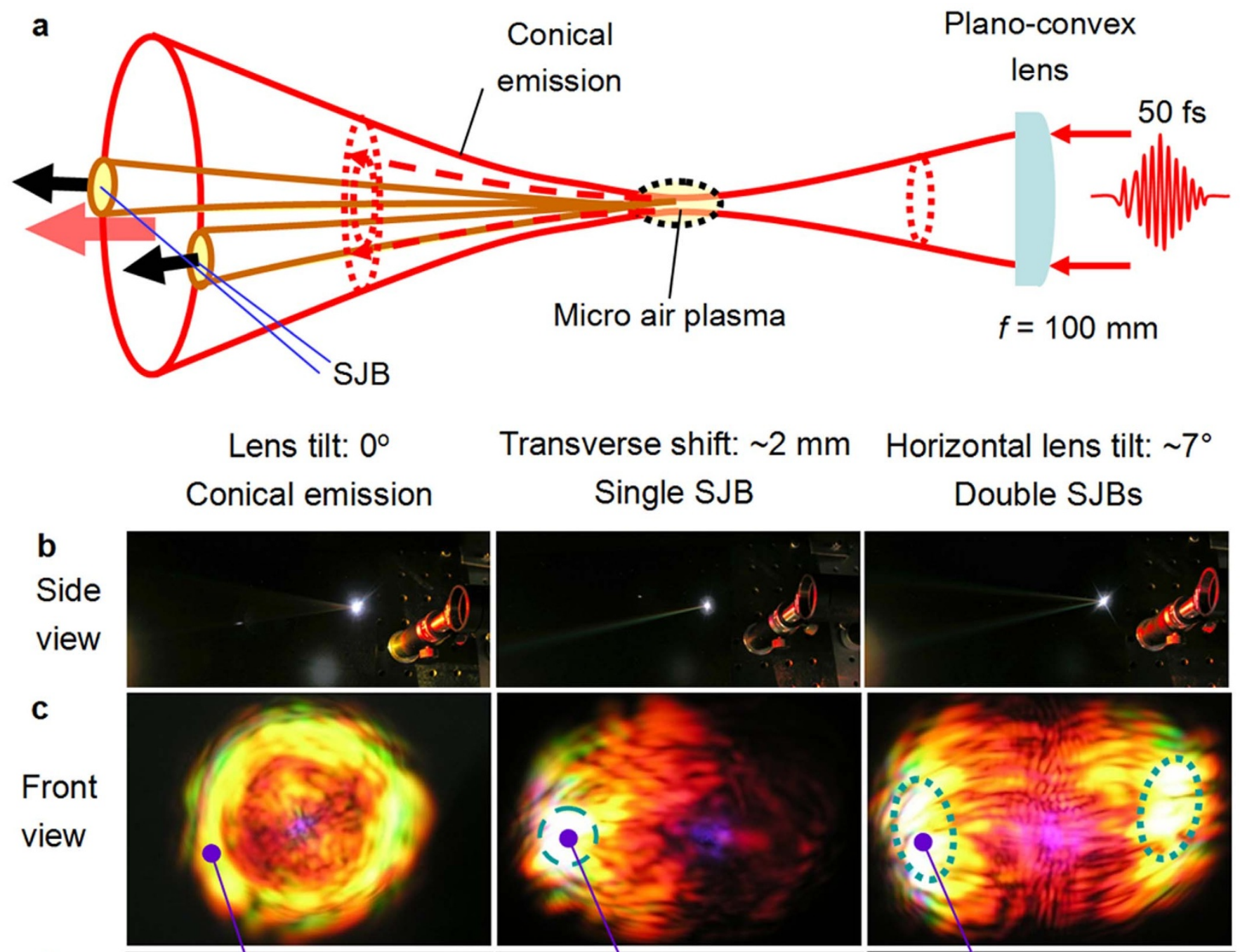

d
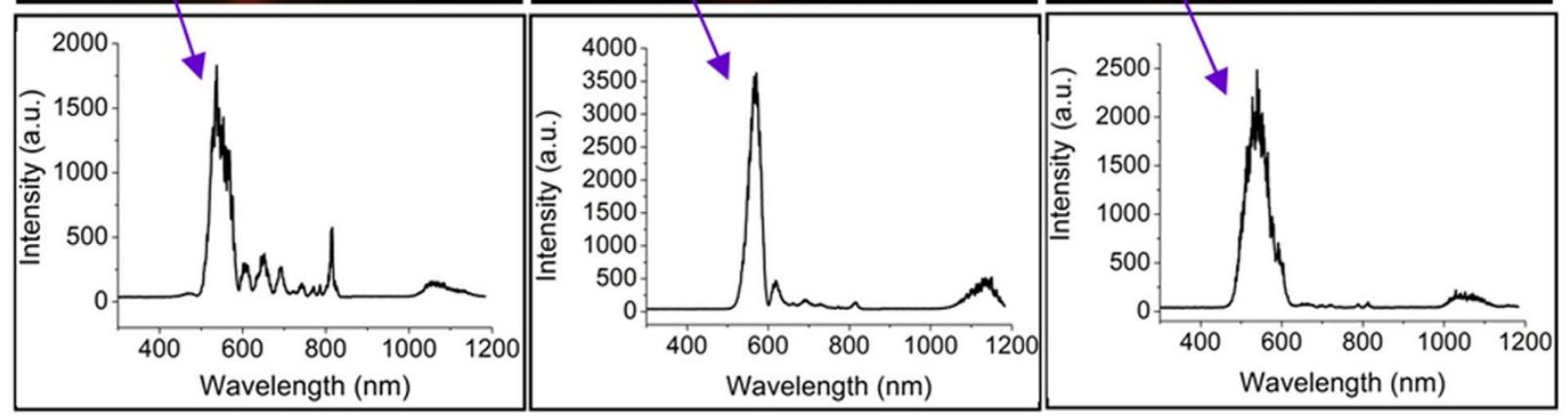

Figure 1 Super-luminescent jet beams (SJBs) generation and the difference \& link between the conical emission (CE) and single (middle column) and double (right column) SJBs. (a) Schematic diagram of the SJB generation. If the generation of the micro plasma is of good rotational or cylindrical symmetry like in the case of $0^{\circ}$ lens tilt, a fairy circular CE beam will be expected. As the symmetry is disrupted via lens transverse shift or tilt, single or double SJBs are produced. (b) Side view pictures of CE and SJBs. (c) Cross sections of the transmitted beam patterns for CE and SJBs after passing through the focal point. (d) Respective spectra measured at the indicated locations of CE and jet beams. The exhibited spectra of the nearly same spectral features show that four-wave mixing is most likely the primary nonlinear process that contributes to the specific color of CE and SJBs.

incident beam (namely there is neither lens tilt nor transverse shift), and a fairy circular conical emission beam will thus be expected. From this reference point a small transverse shift of the lens leads to the generation of a single jet beam and a slight and proper lens tilt can result in the double jet beams which are as indicated by the two narrow cone beams on the left of Fig. 1a. Note that in our experiments the central axis of the focal lens is tilted always horizontally, and correspondingly the observed double superluminescent jet beams also appear in the horizontal plane. Nevertheless, it is verified that the two jet beams will occur in the vertical plane if the direction of the lens axis tilt is made in the vertical direction.

It is found that the SJBs can be observed for 50 fs laser pulses only when the incident excitation power (measured right before the focal lens) is above $0.8 \mathrm{~W}$. The split angle of the double SJBs (Fig. 1b right) increases from $15^{\circ}$ to $16^{\circ}$ when the pump power is raised from $0.8 \mathrm{~W}$ to $2.3 \mathrm{~W}$. In addition to the laser power, pulse duration also affects the SJBs generation. For example, at $2 \mathrm{~W}$ average power, when the laser pulses are frequency-chirped and close to $0.5 \mathrm{ps}$, the jet beams are no longer observed. Compared with relatively stronger/weaker 
focusing lens with $50 \mathrm{~mm} / 150 \mathrm{~mm}$ focal length, the lens of $100 \mathrm{~mm}$ focal length leads to the best results in terms of brightness and clearness of SJBs observed. All the results given in this paper are obtained with the focal lens of $100 \mathrm{~mm}$ focal length.

Characteristics of super-luminescent jet beams. The SJBs ejected from the micro air plasma are clearly shown in the side view pictures of SJBs in Fig. 1b, which are captured by an ordinary color digital camera (C5050Z, Olympus) (For enlarged single and double SJBs see Supplementary Fig. S1). The cross sections of the transmitted beam patterns for CE and SJBs after passing through the focal point are compared in Fig. 1c. These three pictures are obtained by intercepting the transmitted laser beams with a piece of white paper placed 1 meter away from the focal region. It can be seen that for $0^{\circ}$ lens tilt, the recorded beam cross section is approximately circular with strong glowing yellowish area around its perimeter. (In this case when a piece of white paper is used to intercept sideways the transmitting light, the typical cone-shaped light emission is captured (Supplementary Fig. S2d)). Presence of the single/double jet beams is also evidenced by the corresponding single/double bright spots marked with the closed dot lines in the middle and right pictures of Fig. 1c. In the case of single SJB, the position of the bright and yellowish spot is always determined by the direction of the focal lens shift. In particular, if the lens shifts towards left/right by approximately $2 \mathrm{~mm}$, the single jet beam will appear on the left/right side of the disrupted conical emission. For the double jet beams, however, they always appear nearly symmetrically distributed in relative to the central axis when the angle of lens tilt is near 7 degree. The "cerebral sulci and gyri" like beam profiles associated with single/double SJBs shown in the middle/ right pictures in Fig. 1c are dramatically different from that of corresponding normal conical emission (the left picture in Fig. 1c) in terms of the shape regularity and the spatial distribution of intensity profile.

Respective spectra measured at the indicated locations of CE and jet beams are reproduced in Fig. 1d. Distinctive spectral features at $\sim 520 \mathrm{~nm}$ and $\sim 1040 \mathrm{~nm}$ are present in the all three traces, which imply that an intrinsic connection exists between the CE and SJBs. As it will be discussed later, four-wave mixing is most likely the primary nonlinear process that contributes to the specific color of $\mathrm{CE}$ and SJBs.

As it is depicted in Fig. 1a the jet beams themselves have a diverging angle, which is determined to be 1.5 degrees. Such divergence may be controlled or corrected with a suitable collimation lens placed after the air plasma. In the case of double SJBs (see the right picture of Fig. 1b) approximately 16 degree separation angle exists between the two SJBs centers, and after proper collimation two narrow and parallel yellowish light beams with little divergence can propagate for a long distance (Supplementary Fig. S2e).

It is also worth mentioning that when looking from sideways the visibility of SJBs either collimated or not is in fact dependent on the viewing angle in reference with the polarization direction of the linearly polarized femtosecond laser beam. The highest visibility occurs when the plane formed by the viewing direction and the SJBs is normal to the input laser polarization direction, whereas the lowest visibility exists when the viewing direction is parallel to the polarization direction. Experimentally, when rotating a half-wave plate inserted in the incident laser beam path, the visibility of SJBs varies periodically with $\sim 80 \%$ contrast ratio for a fixed observation angle (Supplementary Fig. S3).

It should be stressed that these jet-like beams of light are not optical filaments (OFs). In addition to the significant differences between SJBs and the conventional optical filaments in terms of overall nonlinear interaction strength and interaction length, they also differ at least in the following three aspects: firstly, OFs normally do not exist beyond the plasma ball of higher electron density if both optical filament and plasma ball are present ${ }^{19}$, and also as implied by their names OFs do not diverge for a relatively long distance, whereas SJBs diverge from their starting points; secondly, a single OF is essentially a self-trapped pump beam accompanied by a large area of energy reservoir and a low density plasma channel, but SJBs originate from the surface of the high density plasma ball (See details in Discussion in the following) and thus deviate from the input excitation beam at the very beginning; thirdly, viewed sideways, OFs always appear whitish while the SJBs after collimation are more like colored beams. A more detailed comparison of major characteristics of SJBs and OFs can be found in Supplementary Table S1.

Spectral measurements. To help understand the key characteristics of the SJBs, spectral measurements are performed at four different locations along the propagating light beam(s) as marked by the letters A, B, C and D in Fig. 2a, and the measured corresponding spectra are shown in Fig. 2b. Here, the double SJBs generated with $50 \mathrm{fs}$ pulses at $2 \mathrm{~W}$ average power are collimated with a doubleconvex lens of $75 \mathrm{~mm}$ focal length.

In Fig. 2b, trace A represents the spectrum of the scattered femtosecond pump laser pulses. The emission spectrum of the micro plasma at the focus is shown by trace $\mathrm{B}$, whose characteristics have been studied elsewhere ${ }^{20,21}$. Trace $C$ shown in purpose in the same plot with trace A presents the typical sideways emission (See Methods) spectrum of the collimated jet beam. This blue-shifted spectrum (up to approximately $550 \mathrm{~nm}$ ) implies the existence of certain degree of air ionization along the collimated SJB path. The spectrum of the collimated SJB that has a brilliant yellowish color is shown by trace $\mathrm{D}$, where two distinctive spectral bands with the respective central wavelengths of approximately $547 \mathrm{~nm}$ and $1064 \mathrm{~nm}$ are clearly observed. Such a spectral distribution provides us with a good evidence of degenerate four-wave mixing ${ }^{22}$ while experimental recording of the Stokes waves (around $1064 \mathrm{~nm}$ ) has not been reported in the previous studies in this regard ${ }^{23}$. It is pictured that the initial excitation laser beam with a central wavelength around $800 \mathrm{~nm}$ strongly ionizes the air molecules in the focal region and in the meantime generates large amount of blue-shifted supercontinuum because of strong self-steepening effect ${ }^{24}$. (For more spectral examples taken at various locations across the nonlinearly diffracted laser beams, see Supplementary Fig. S4). It is such blue shifted spectral component that functions as the pump for four-wave mixing under the right phase-matching condition. This result is in a good analogy with the so-called self-phase modulation (SPM) initiated four-wave mixing observed inside optical fibers in previous experiments ${ }^{25}$.

Two dimensional contour plots of far-field angular spectra may be constructed from the spectral traces measured along the central horizontal line of the diffracted beams that encompass single or double super-luminescent jet beams, as well as many colored speckles (Supplementary Fig. S5). Such angular spectra plots can show not only the existence of distinct Stokes wave in a more dramatic way, but also their great difference from the reported X-waves ${ }^{26}$.

\section{Discussion}

For the relatively tight focusing adopted in our experiments, intensity clamping $^{14}$ as a result of dynamic balance between self-focusing and plasma defocusing does not apply. According to the following numerical simulations and our experimental conditions, the superbright micro plasma ball near the focal point implies that the actual peak intensity of the light beam must be extremely high such that the air ionization may reach saturation. Thus, in contrast to the situations of filament formation where relatively lower intensity $\left(10^{13}-10^{14} \mathrm{~W} / \mathrm{cm}^{2}\right)$ and longer interaction length exist, in our case substantially higher peak intensity $\left(10^{15}-10^{16} \mathrm{~W} / \mathrm{cm}^{2}\right)$ and very short interaction length are present.

The evolution of free electron density inside the laser-induced air plasma is simulated following the method used peviously ${ }^{27,28}$, and the 


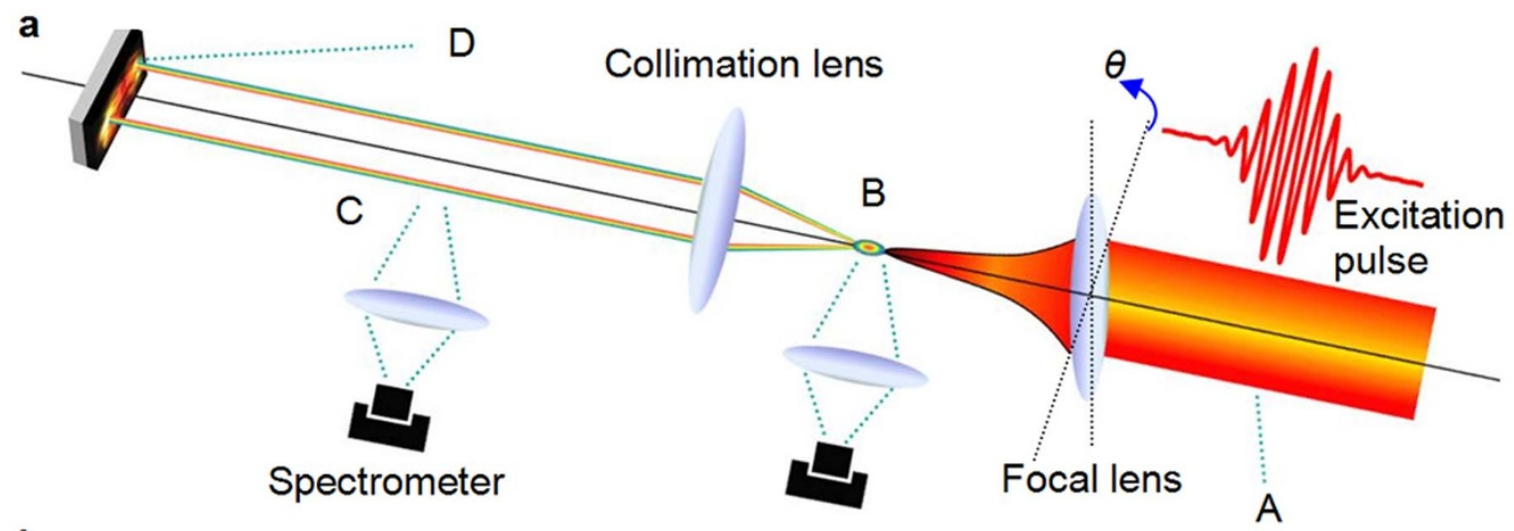

b
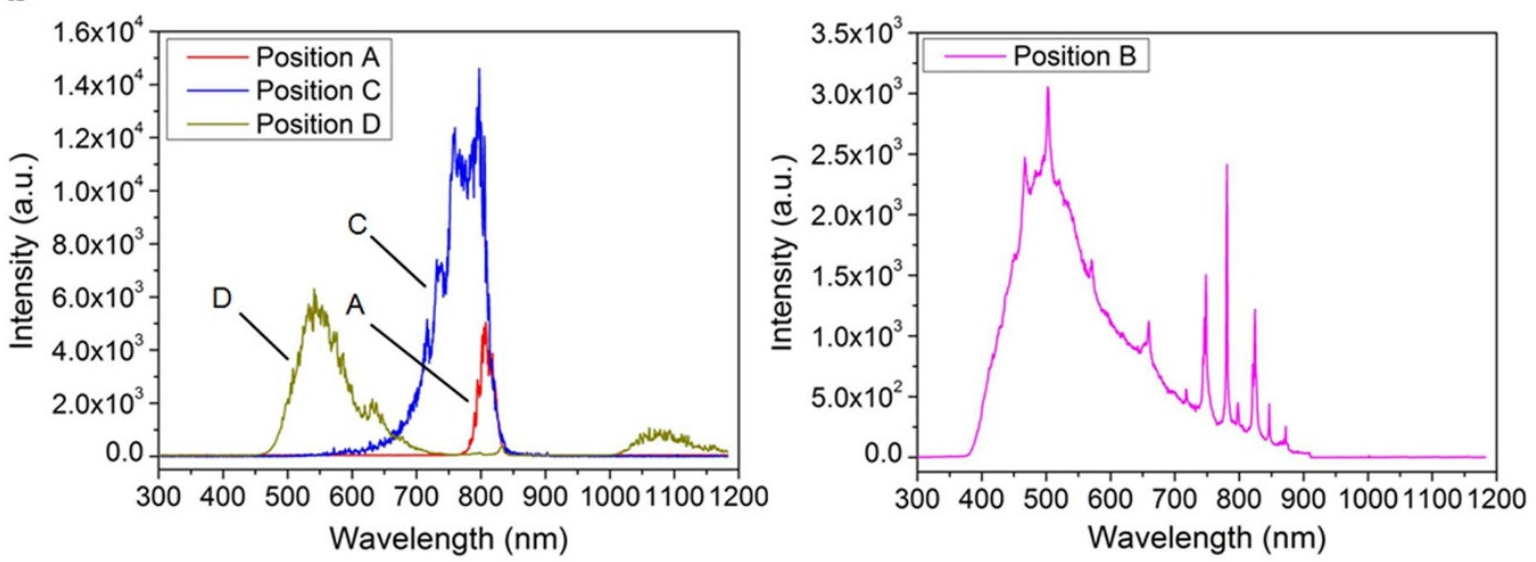

Figure $2 \mid$ Characteristic spectra in the super-luminescent jet beam generation. (a) Schematic diagram (top view) of double SJBs generation and the four spectral check points respectively labeled as A, B, C, and D along the propagation beam. (b) Spectra measured at the respective positions A, B, C, and D indicated in (a) for vertically polarized $50 \mathrm{fs}$ excitation pulses with single pulse energy of $2 \mathrm{~mJ}$. Trace A: the excitation laser pulse spectrum (red line); Trace B: the emission spectrum of the micro air plasma at the focus (magenta line); Trace C: the sideways emission spectrum from one of the collimated double jet beams (blue line); Trace D: forward emission of the propagating yellowish jet beam (dark yellow line).

simulation results pertinent to our experiments are shown in Fig. 3. Note that in obtaining the results given in Fig. 3, multiphoton/tunneling ionization is considered for intensity below/above $6 \times$ $10^{13} \mathrm{~W} / \mathrm{cm}^{2}$ respectively. (The red star in Fig. 3a marks the beginning of tunneling ionization). We have also assumed that the ionization process is halted as soon as the electron density reaches the neutral molecule density in air $\left(\rho_{e o}=2.69 \times 10^{19} \mathrm{~cm}^{-3}\right)$. (Although double charge ionization is possible in practice, this will not affect the electron density in an order of magnitude). As shown in Fig. 3a, for the given pulse intensity the electron density reaches saturation at the time well before the arrival of the pulse peak and remains saturated for the rest majority part of the pulse.

Fig. $3 \mathrm{~b}$ shows the calculated spatial distribution of the refractive index of air plasma associated with the same set of input laser parameters as those used above. Apparently, there exists a saturation region in the central focal area where the refractive index of plasma becomes constant. The longitudinal length of this saturation region is close to $1.2 \mathrm{~mm}$, which is about ten times the Rayleigh range. And the maximum lateral length of this region is about $27 \mu \mathrm{m}$, nearly four times the radius of the beam waist.

With the properties of air plasma known, we can now better understand the nonlinear divergence of conical emission. Compared with the case of linear diffraction (without plasma generation), the observed conical emission has a much larger divergence angle (Supplementary Fig. S6), and this phenomenon may be qualitatively explained by the refraction at the plasma-air interface of the saturated plasma zone, namely, the so-called surface model of conical emission ${ }^{29}$. For example, assuming an incident ray at the plasma-air interface with an oblique angle $\theta_{d}$, the corresponding refractive ray leaving the plasma shell into the air will have an oblique angle $\theta_{n}$ that can be estimated from the simple relation of $n_{p} \cos \theta_{d}=n_{a} \cos \theta_{n}\left(n_{a}, n_{p}\right.$ are the refractive indices of air and plasma, respectively). From our simulation, we have $n_{p} \approx 0.9923, n_{a}=1.00027$, and with these values we obtain $\theta_{n} \approx$ $7.9^{\circ}$ for $\theta_{d} \approx 3.2^{\circ}\left(\right.$ at $\left.1 / \mathrm{e}^{2}\right)$, which appears in reasonably good agreement with the experimental observation. However, in this rather simplified model we have not taken account of the dispersion effect, which will attribute to a substantial color separation.

Understandably, to analyze four-wave mixing that occurs at the periphery of the air plasma zone, an effective refractive index $n(\lambda)$ needs to be considered, which should be a mixture of refractive indices of ambient air and air plasma. (For air, Kerr-effect specified by the product of nonlinear refractive index coefficient $n_{2}$ and the peak intensity $I$ should also be included). We thus have the following approximate expression:

$$
n(\lambda) \approx\left(n_{a}+n_{2} I\right)\left(1-\frac{\rho_{e}}{\rho_{e o}}\right)+n_{p} \frac{\rho_{e}}{\rho_{e o}} .
$$

The first and the second items on the right hand side of Eq. (1) represent the corresponding contributions from air and plasma. From Eq. (1), it is clear that the ionization ratio $\rho_{e} / \rho_{e o}$ plays a critical role in the determination of $n(\lambda)$. If $\rho_{e} / \rho_{e o}=1$, it is $100 \%$ ionized, we have $n(\lambda)=n_{p}$. If $\rho_{e} / \rho_{e o}=0$, no air ionization exists and thus $n(\lambda)=$ $n_{a}+n_{2} I$. It should be noted that the electron density $\rho_{e}$ is also a nonlinear function of light intensity ${ }^{30}$.

The dispersion feature of $n(\lambda)$ can be found out by the individual dispersion property of air and plasma, which may be described by $n_{a}(\lambda)=1+77.6\left(1+7.52 \times 10^{-3} / \lambda^{2}\right)\left(P_{a} / T\right) \times 10^{-6}$ and $n_{p}(\lambda)=(1$ 

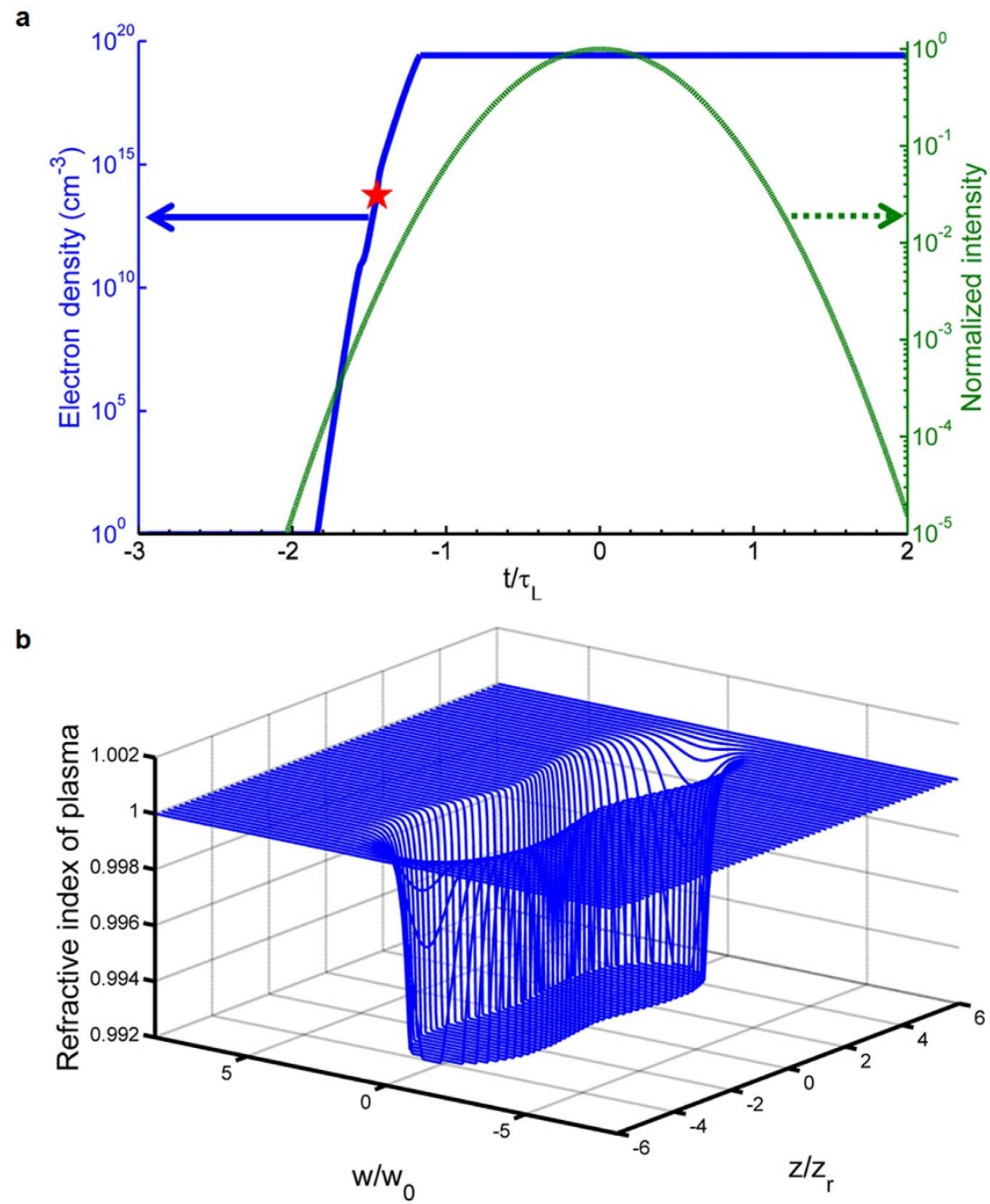

Figure 3 Simulation results of electron density and refractive index distribution of laser-induced air plasma. (a) Calculated free electron density (blue solid line) during the $50 \mathrm{fs}$ laser pulse (green dash line) ionization at the focal point. The laser intensity axis (right column) and the time axis are respectively normalized to the peak intensity $\left(2.6 \times 10^{16} \mathrm{~W} / \mathrm{cm}^{2}\right)$ and the laser pulse width $\left(\tau_{\mathrm{L}}\right)$. The red star on the rising edge marks the switching point from multi-photon ionization to tunneling ionization. (b) Simulation results of the refractive index distribution for the air plasma generated by focused $2 \mathrm{~mJ}, 50 \mathrm{fs}$ laser pulses with the $100 \mathrm{~mm}$ focal length lens. Both transverse and propagation axes are respectively normalized to the radius of beam waist $\mathrm{w}_{0}$ and the corresponding Rayleigh range $z_{\mathrm{r}}$.

$\left.-\rho_{e}(r, z, t) / \rho_{c r}\right)^{1 / 2}$ respectively. Here $P_{a}$ is the air pressure, $T$ the temperature, $\rho_{c r}$ the critical electron density. Both $\rho_{e}$ and $\rho_{c r}$ are wavelength dependent. In our case, while air ionization inside the air plasma zone mostly reaches saturation as evidenced in Fig. 3b, around the plasma-air border, however, air is very likely only partially ionized. In Fig. 4 a the predicted group velocity dispersion of the micro air plasma for $\rho_{e} / \rho_{e o}=0.04$ is obtained on the basis of Eq. (1).

It is known that for degenerate four-wave mixing the following energy and momentum conservation conditions hold ${ }^{22,31}$ :

$$
\left\{\begin{array}{c}
2 \omega_{p}=\omega_{s}+\omega_{a s} \\
2 k_{p}=k_{s}+k_{a s}
\end{array}\right.
$$

where $\omega_{i}$ and $k_{i}(i=p, s, a s)$ denote respectively the angular frequencies and propagation constants of the pump, Stokes and anti-Stokes waves, and $k_{i}=\left(2 \pi / \lambda_{i}\right) n\left(\lambda_{i}\right)$ with $n\left(\lambda_{i}\right)$ governed by Eq. (1). As the pump, Stokes and anti-Stokes waves' wave vectors are all assumed to be parallel to each other, the momentum conservation in Eq. (2) is expressed by an algebraic equation. For a given ionization ratio, Eq. (2) can be solved for different pump wavelengths, resulting in the socalled phase-matching curves. In Fig. $4 \mathrm{~b}$ a pair of such curves are obtained for $\rho_{e} / \rho_{e o}=0.04$. It can be found that the data points of $547 \mathrm{~nm}$ and $1064 \mathrm{~nm}$ wavelengths are in good agreement with the experimentally recorded FWM spectrum in Fig. 2b. Corresponding to these predicted Stokes and anti-Stokes waves, a pump wavelength of $722 \mathrm{~nm}$ is derived. Experimentally the pump wavelength transformation from $800 \mathrm{~nm}$ to $722 \mathrm{~nm}$ must be caused by the other nonlinear effect(s). At this point we also cannot derive and thus explain why the ionization ratio is $4 \%$. Instead, the ionization ratio is used as an adjustable parameter in our calculation so that phasematching can be achieved at the wavelengths consistent with the experimental observation.

Now let us discuss briefly how the lens shift or lens tilt leads to SJBs generation. In the case of normal incidence, the entire air ionization process is circularly symmetrical along the propagation axis, so does the effective refractive index given in Eq. (1). In other words, phasematching condition by the second expression of Eq. (2) is met everywhere on the surface of conical emission cone. This conforms to the measured circular yellowish color band of FWM in the CE cross section. When the focal lens is transversely shifted, however, the cylindrical symmetry of the focused beam intensity is then broken, 
a
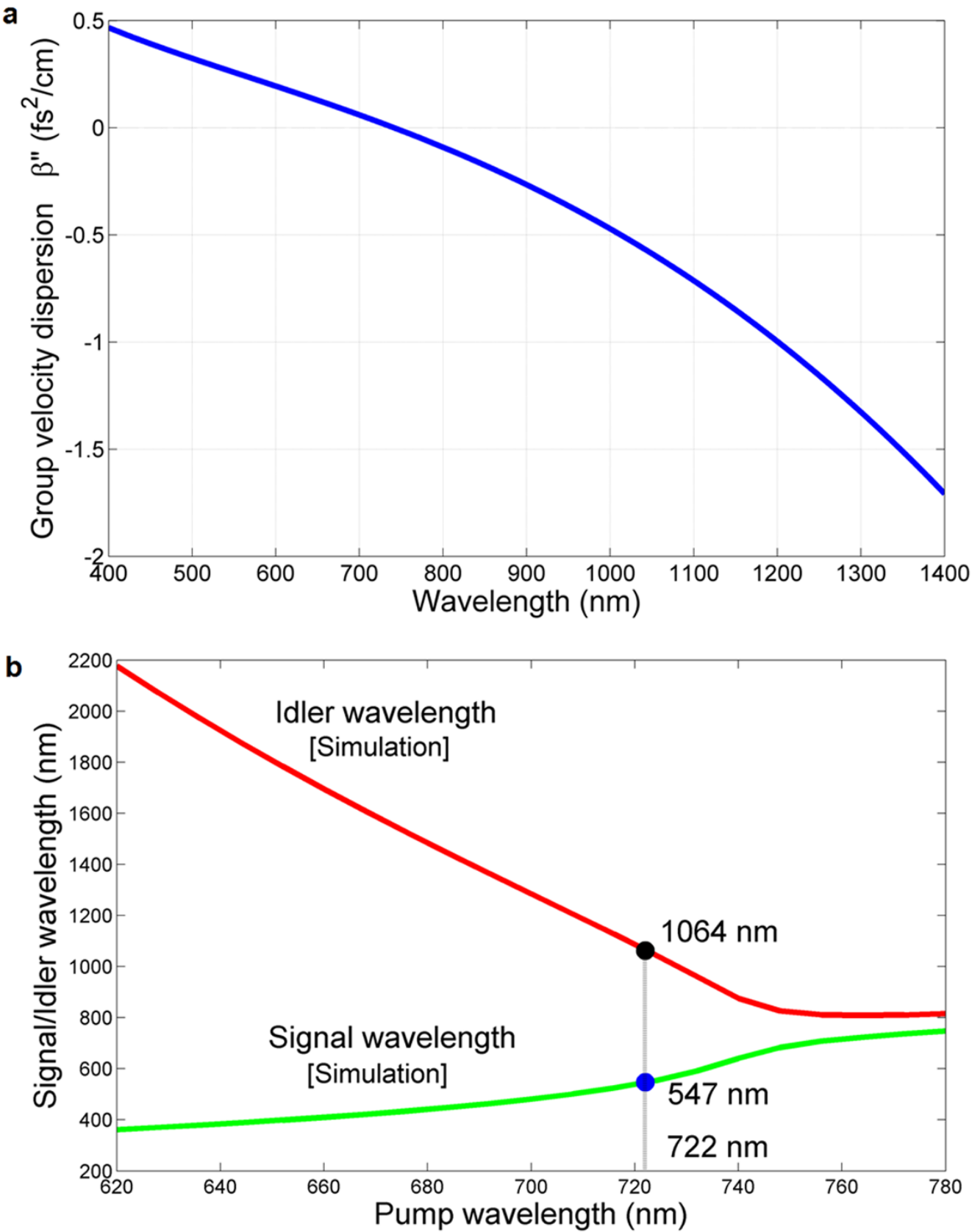

Figure $4 \mid$ Dispersive property and four-wave mixing conditions of the laser-induced air plasma. (a) Wavelength dependence of the group velocity dispersion for the ionized air with an ionization ratio of $4 \%$. (b) Calculated nonlinear phase-matching diagram for the four-wave mixing process taking place in the single/double SJBs and CE cone surface.

and thus the phase-matching condition is met only at the specific direction. Particularly, when the focal lens shifts towards one side, the direction of higher intensity ray also deflects towards the same side where the single SJB appears. When the lens is properly tilted, say in the horizontal plane around its central axis, the astigmatism is introduced in the focused beam, i.e., the focus in the tangential plane (also the horizontal plane) becomes shorter than that in sagittal plane, and in the meantime, the rays in tangential plane will arrive at their focal positions ahead of the sagittal plane rays which is confirmed experimentally using a time-resolved shadowgraphic apparatus ${ }^{15}$. This means that air plasma is generated by the tangential plane rays before the sagittal rays reach their focus. And spatially, this results in relatively stronger air ionization in the horizontal plane than those in the sagittal plane and so the intensity dependent phase-matching can be only achieved in the tangential or the horizontal plane.
In summary, colored super-luminescent jet beams (SJBs) are experimentally observed emanating from the micro air plasma ignited by focused 50 femtosecond laser pulses of millijoule-level pulse energy. Such jet-like light beam(s) of slight divergence, being formed at the cone surface of substantially distorted conical emission, differ from the conventional white light filaments. Sufficiently but not too tight focusing and proper shift or tilt of the focal lens are critical in generating single or double SJBs. Short pulse duration is also important in terms of having high enough peak intensity so that strong nonlinear effect like self-steepening and photo-ionization saturation can coexist for a relatively short interaction length. Spectral measurements show that four-wave mixing at specific diffraction angle(s) at the cone surface of disrupted conical emission is responsible for the observed phenomenon. Once being collimated, these brilliantly-colored jet beams with better coherence than the white 
light from optical filaments can propagate over a long distance in air and very likely they may find some applications in remote sensing in future.

\section{Methods}

Experimental setup. A commercial $1 \mathrm{kHz}$ Ti: sapphire femtosecond laser amplifier system (Integra-HE, Quantronix Inc.) is employed in our experiments to produce the $800 \mathrm{~nm}$ laser pulses with pulse duration tunable from 50 fs to 16 ps which may be conveniently obtained by misaligning the grating separation in pulse compressor within the femtosecond laser amplifier system and single pulse energy up to $6 \mathrm{~mJ}$. The femtosecond laser pulses from the amplifier system is vertically polarized and focused directly in air after being reflected by two $45^{\circ}$ broadband HR dielectric mirrors. The focal lens of $100 \mathrm{~mm}$ focal length is an AR-coated single plano-convex lens, having one-inch diameter and always with its curved surface facing the incident beam. To generate the super-luminescent jet beams (SJBs) the femtosecond laser pulses is first aligned passing through the focal lens at normal incidence, by which the brightest micro air plasma is obtained. Single/double SJB(s) (such as those shown in Fig. 1b) can be then routinely produced by properly shifting/tilting the focal lens. Slight tilting of the lens is made always through gently rotating the lens mount in the horizontal plane.

Analyses of spatial beam profiles. To examine the spatial features of SJBs, a piece of carton paper is used to partially intercept sideways the propagating beam after the focal point so that side view pictures like that in Supplementary Fig. S2d can be obtained. To find out the spatial power distribution of the nonlinearly diffracted beam, a chessboard-like mask of alternative square holes is placed in front of the largely diffracted beam spot and right before the detector head of an optical power meter. Optical power at each hole is then measured with the power meter (NOVA II, Ophir Inc.). From these measurements, an approximate power conversion ratio of $8.8 \%$ (or $13.3 \%$ ) for the single (or double) $\mathrm{SJB}(\mathrm{s}$ ) is determined. Although the transmitted pump beam in the near infrared (around $800 \mathrm{~nm}$ ) still dominates the overall power distribution especially in the central area of the beam profile, SJBs are more pronounced in the visible spectral region. In other words, SJBs occupy a significant portion among the laser produced supercontinuum. Such a feature is verified by inserting in front of the power meter a short-pass filter (650FL07-25, Andover) that rejects the pump wavelength and has high transmission for the visible spectrum below $700 \mathrm{~nm}$.

Spectral measurements. In spectral measurements, at location C in Fig. 2a the scattered light from one section of the collimated double jet beams (or the sideways emission) is coupled into a Shamrock spectrometer (Shamrock SR-750, Andor) equipped with an ICCD detector (DH740i-18U-03, iStar, Andor). In this case, in addition to the imaging lens, a couple of steering mirrors (not shown in Fig. 2a) are employed for rotating the sampled source section by 90 -degree so that the image becomes parallel to the entrance slit of the spectrometer and so improved light coupling efficiency is achieved. Spectral measurements at the other three locations labeled by A, B and D respectively are made with a miniature fiber-pigtailed spectrometer (Model S2000, Ocean Optics) of lower sensitivity but more convenience of operation. In measuring the spectrum of the collimated jet beams (not the scattered light) at location D, three optical wedges are utilized to reflect off the beam three times and thus significantly reduce the optical power entering the spectrometer. It should be noted that for all the spectral traces given in this paper the spectral response of the detection system has not been corrected, and thus the relative heights of the spectral peaks, especially those in Fig. 1d, trace D in Fig. $2 b$ do not present the real signal strength.

1. Marburger, J. H. Self-focusing: theory. Prog. Quantum Electron. 4, 35-110 (1975).

2. Brodeur, A. \& Chin, S. L. Ultrafast white-light continuum generation and selffocusing in transparent condensed media. J. Opt. Soc. Am. B 16, 637-650 (1999).

3. Chin, S. L., Brodeur, A., Petit, S., Kosareva, O. G. \& Kandidov, V. P. Filamentation and supercontinuum generation during the propagation of powerful ultrashort laser pulses in optical media (white light laser). J. Nonlinear Opt. Phys. Mat. 8, 121-146 (1999).

4. Nibbering, E. T. et al. Conical emission from self guided femtosecond pulses in air. Opt. Lett. 21, 62-64 (1996)

5. Kelley, P. L. Self-focusing of optical beams. Phys. Rev. Lett. 15, 1005-1008 (1965).

6. Braun, A. et al. Self-channeling of high-peak-power femtosecond laser pulses in air. Opt. Lett. 20, 73-75 (1995).

7. Couairon, A. \& Mysyrowicz, A. Femtosecond filamentation in transparent media. Phys. Rep. 441, 47-189 (2007).

8. Bergé, L., Skupin, S., Nuter, R., Kasparian, J. \& Wolf, J. P. Ultrashort filaments of light in weakly-ionized, optically-transparent media. Rep. Prog. Phys. 70, 1633-1713 (2007).

9. Tzortzakis, S. et al. Breakup and fusion of self-guided femtosecond light pulses in air. Phys. Rev. Lett. 86, 5470-5473 (2001).

10. Gaeta, A. L. Catastrophic collapse of ultrashort pulses. Phys. Rev. Lett. 84, 3582-3585 (2000).

11. Théberge, F., Aközbek, N., Liu, W. W., Becker, A. \& Chin, S. L. Tunable Ultrashort Laser Pulses Generated through Filamentation in Gases. Phys. Rev. Lett. 97, 023904 (2006)
12. Aközbek, N. et al. Third-harmonic generation and self-channeling in air using high-power femtosecond laser pulses. Phys. Rev. Lett. 89, 143901 (2002).

13. Liu, C. S. \& Tripathi, V. K. Stimulated Raman scattering in a plasma channel. Phys. Plasmas 3, 3410 (1996).

14. Kasparian, J., Sauerbrey, R. \& Chin, S. L. The critical laser intensity of self-guided light filaments in air. Appl. Phys. B 71, 877-879 (2000).

15. Zhang, N., Zhu, X. N., Yang, J. J., Wang, X. L. \& Wang, M. W. Time-resolved shadowgraphs of material ejection in intense femtosecond laser ablation of aluminum. Phys. Rev. Lett. 99, 167602 (2007).

16. Gattass, R. R. \& Mazur, E. Femtosecond laser micromachining in transparent materials. Nature Photon. 2, 219-225 (2008).

17. Xu, Z. J., Yu, Y., Zhang, N., Zhao, J. F. \& Zhu, X. N. Generation of Superluminescent Jet Light through Disrupted Conical Emission. CLEO: 2013: Laser Filamentation (QW1E), San Jose. 10.1364/CLEO_QELS.2013.QW1E.5 (2013, June 9-14).

18. Xu, Z. J., Zhu, X. N., Zhang, N. \& Yu, Y. Observation of super-luminescent jet beam from femtosecond laser-induced air plasma. CLEO-PR \& OECC/PS 2013 Kyoto. 10.1109/CLEOPR.2013.6600495 (2013, June 30- July 4).

19. Liu, W. W., Luo, Q. \& Chin, S. L. Competition between multiphoton/tunnel ionization and filamentation induced by powerful femtosecond laser pulses in air. Chin. Opt. Lett. 1, 56-59 (2003).

20. Talebpour, A., Abdel-Fattah, M., Bandrauk, A. D. \& Chin, S. L. Spectroscopy of the gases interacting with intense femtosecond laser pulses. Laser Phys. 11, 68-76 (2001).

21. Zhu, X. N. \& Fu, R. L. Emission spectra of microplasma generated by femtosecond laser pulses. Proc. SPIE 4914: High-Power Lasers and Applications II, Shanghai. 10.1117/12.481796 (2002, September 6).

22. Nodop, D. et al. Efficient high-power generation of visible and mid-infrared light by degenerate four-wave mixing in a large-mode-area photonic-crystal fiber. Opt. Lett. 22, 3499-3501 (2009).

23. Kandidov, V. P. et al. Self-transformation of a powerful femtosecond laser pulse into a white-light laser pulse in bulk optical media (or supercontinuum generation). Appl. Phys. B 77, 149-165 (2003).

24. Yang, G. Z. \& Shen, Y. R. Spectral broadening of ultrashort pulses in a nonlinear medium. Opt. Lett. 9, 510-512 (1984).

25. Zhu, X. N. \& Sibbett, W. Propagation characteristics of femtosecond pulses in erbium-doped monomode optical fibers. IEEE J. Quantum Electron. QE- 27, 101 (1991).

26. Faccio, D. et al. Conical emission, pulse splitting, and X-wave parametric amplification in nonlinear dynamics of ultrashort light pulses. Phys. Rev. Lett. 96, 193901 (2006).

27. Vogel, A., Noack, J., Hüttman, G. \& Paltauf, G. Mechanisms of femtosecond laser surgery of cells and tissues. Appl. Phys. B 81, 1015-1047 (2005).

28. Yu, Y., Xu, Z. J., Zhang, N. \& Zhu, X. N. Characteristics of electron density in air plasma produced by tightly-focused 50 fs laser pulses. CLEO-PR \& OECC/PS 2013, Kyoto. 10.1109/CLEOPR.2013.6600496 (2013, June 30- July 4).

29. Golub, I. Optical characteristics of supercontinuum generation. Opt. Lett. 15 , 305-307 (1990)

30. Feng, Q. et al. Theory and simulation on the threshold of water breakdown induced by focused ultrashort laser pulses. IEEE J. Quantum Electron. QE- 33, 127 (1997).

31. Agrawal, G. P. [Ch. 10 Parametric Processes] Nonlinear Fiber Optics [Kelley, P. L. et al. (ed.)] [389-408] (Academic Press, San Diego, 2001).

\section{Acknowledgments}

The authors acknowledge financial supports from the National Natural Science Foundation of China under Grant Nos. 11274185, 61137001, and 11004111.

\section{Author contributions}

Z.J.X. designed and implemented the experiments. X.N.Z. conceived the idea and the theoretical model for explaining the observed phenomena. Y.Y. carried out all the numerical analysis. N.Z. helped to clarify the dynamic characteristics of laser-induced plasma. J.F.Z took part in the initial experiments. Z.J.X. and X.N.Z. wrote the main manuscript text and Z.J.X. prepared figures 1-4. All authors reviewed the manuscript and contributed to the paper.

\section{Additional information}

Supplementary information accompanies this paper at http://www.nature.com/ scientificreports

Competing financial interests: The authors declare no competing financial interests.

How to cite this article: Xu, Z.J., Zhu, X.N., Yu, Y., Zhang, N. \& Zhao, J.F. Super-luminescent jet light generated by femtosecond laser pulses. Sci. Rep. 4, 3892; DOI:10.1038/srep03892 (2014).

This work is licensed under a Creative Commons AttributionNonCommercial-NoDerivs 3.0 Unported license. To view a copy of this license, visit http://creativecommons.org/licenses/by-nc-nd/3.0 